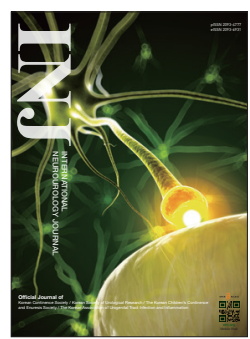

\title{
Underactive Bladder; Review of Progress and Impact From the International CURE-UAB Initiative
}

\author{
Michael B. Chancellor, Sarah N. Bartolone, Laura E. Lamb, Elijah Ward, Bernadette M.M. Zwaans, Ananias Diokno \\ Department of Urology, Beaumont Health System, Royal Oak, MI, USA
}

\begin{abstract}
There is a significant need for research and understanding of underactive bladder (UAB). The International Congress of Urologic Research and Education on Aging UnderActive Bladder (CURE-UAB) was organized by Doctors Michael Chancellor and Ananias Diokno in order to address these concerns. CURE-UAB was supported, in part, by the US National Institute of Aging and National Institute of Diabetes Digestive and Kidney. Since 2014, there have been 5 successful CURE-UAB congresses. They have brought together diverse stakeholders in the UAB field to identify areas of major scientific challenge and initiated a call to action among the medical community. In this review, we will highlight current and novel treatments under development for $\mathrm{UAB}$ and the progress and impact from the CURE-UAB initiative.
\end{abstract}

Keywords: Underactive urinary bladder; Overactive urinary bladder; Urodynamics; Sarcopenia; Aging

- Fund/Grant Support: Funding support was in parts sponsored by the NIA, NIDDK (R13AG047010) and the Aikens Center for Neurourology Research at Beaumont Health System (Royal Oak, MI, USA).

- Conflict of Interest: Michael B Chancellor is one of the inventors of the autologous muscle-derived cell process and has received royalty payments for the stem cell process and payments for consulting from Cook MyoSite. Except for that, no potential conflict of interest relevant to this article was reported.

\section{INTRODUCTION}

Underactive bladder (UAB) is a complex constellation of symptoms with few treatment options that can significantly diminish the quality-of-life (QoL) of those affected. Many UAB patients feel helpless when they cannot void or are unable to adequately empty their bladder. UAB is prevalent, underdiagnosed, and represents an unmet medical need in elderly and other populations [1]. There are no outcome-validated effective therapeutics for UAB and research on promising candidates has slowed due to a lack of consensus and understanding regarding its diagnosis and treatment [2].

While treatments for overactive bladder $(\mathrm{OAB})$ have contin- ued to improve, there are still no effective treatments for patients with this converse condition. UAB symptoms can include straining, incomplete bladder emptying, hesitancy, frequent urination, or leakage due to overflow incontinence. UAB can be caused by myogenic and neurogenic conditions, as well as aging and medication side effects.

The current management of $\mathrm{UAB}$ treatment for urinary retention is clean intermittent catheterization (CIC) or indwelling catheters to empty the bladder [3]. However, complications and adverse effects can arise, especially during long-term use. Current research and treatment under consideration for UAB are described in detail in previous summary papers from Congress of Urologic Research and Education on Aging UnderActive

Corresponding author: Michael B. Chancellor (iD https://orcid.org/0000-0001-9480-8972 Department of Urology, Beaumont Health System, 3811 West 13 Mile Road, Royal Oak, MI 48073, USA

E-mail: michael.chancellor@beaumont.org

Submitted: January 11, 2020 / Accepted after revision: January 29, 2020 
Bladder (CURE-UAB) [4,5].

\section{THE INTERNATIONAL CURE-UAB}

The purpose of the CURE-UAB conference series was to gather diverse stakeholders from multiple disciplines and backgrounds together to address the unmet needs of $U A B$ research and care. The first CURE-UAB, sponsored by the National Institute on Aging (NIA), National Institute of Diabetes and Digestive and Kidney Diseases (NIDDK), Aikens Center for Neurourology Research at Beaumont Health System, and The Underactive Bladder Foundation was held in February 2014 in Washington, DC. The second international meeting was December 2015 in Denver, CO; the third meeting was held in March 2017 in Washington, DC; the fourth meeting was held in November 2018 in Honolulu, HI; and the fifth meeting was held in December 2019 in East Lansing, MI.

\section{Public, Academic and Industry Stakeholders}

CURE-UAB has received support from the following academic and public stakeholders: The Aikens Center for Neurourology Research, American Urological Association, Underactive Bladder Foundation (https://www.underactivebladder.org), Geriatric Urology Society (GUS), and Society of Urology Nursing Association (SUNA). Additional support and cosponsorships from public, academic and industry stakeholders significantly added to the international congresses. The UAB Foundation is a patient advocacy group dedicated to encouraging, supporting, and monitoring action on the implementation of evidence-based efforts to promote global action on UAB. The UAB Foundation focuses on involving key stakeholders and representing $\mathrm{UAB}$ patients and their family and friends who care deeply about this underrepresented disease. American Urological Association (AUA), GUS, and SUNA are professional organizations for clinicians and researchers in the urology fields.

\section{RETURN ON INVESTMENT OF THE INTERNATIONAL COLLABORATIVE CURE-UAB GRANT}

The United States National Institutes of Health (NIH) R13 grant mechanism supports high-quality scientific conferences to promote unmet medical needs, awareness, and engage public and private stakeholders. Starting in 2014, the NIA and NIDDK funded the R13 (R13AG047010) grant entitled CURE-UAB to bring stakeholders together to educate and promote awareness on the topic of UAB. We hereby report metric of the impact of this R13 on the medical and scientific community.

A review of the impact on science, public awareness, and research advances were completed by comparing publications and grants from the time-period prior to the 2014 R13 award and the 5 years since R13 funding.

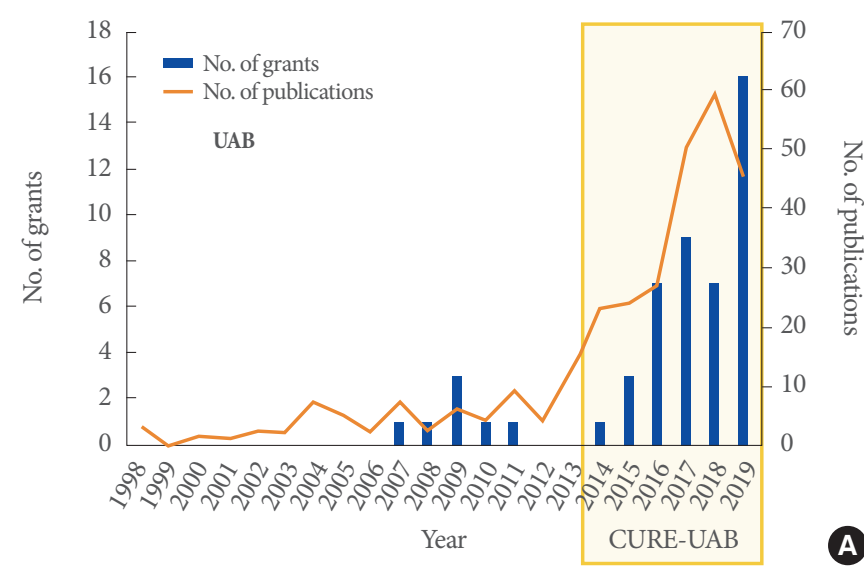

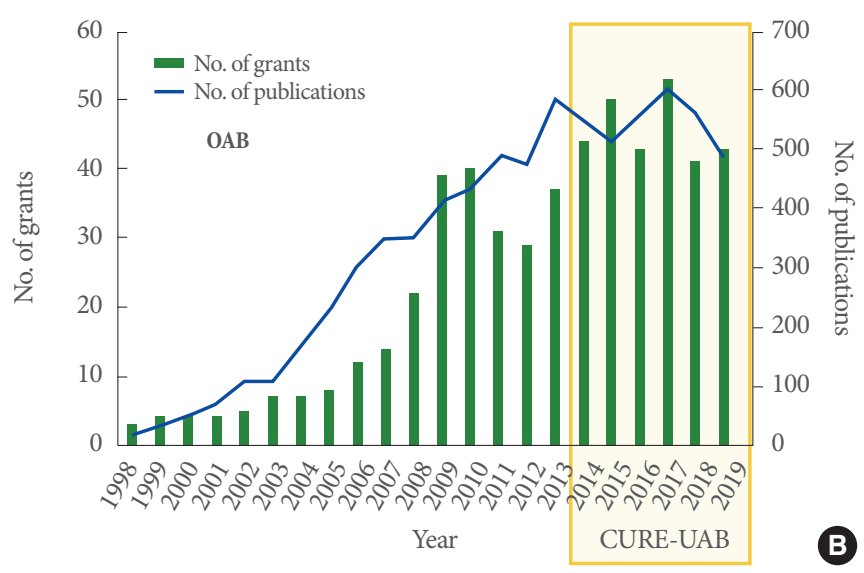

Fig. 1. The number of publications and grants on underactive bladder (UAB, A) and overactive bladder $(\mathrm{OAB}, \mathrm{B})$ over time (shown is 1998 to 2019). The CURE-UAB conference series started in 2014 and ended in 2019 (yellow box and shading). Publication count (UAB: orange line, right $y$-axis; OAB: blue line, right $y$-axis) is from PubMed.gov search of "underactive bladder" or "overactive bladder" on 12/17/19. Prior to 1998, there are only 38 publications total for underactive bladder, with the earliest one from 1980. Grant count (UAB: blue bars, left $y$-axis; OAB: green bars, left $y$-axis) is from Grantome.com search of "underactive bladder" or "overactive bladder" on 12/17/19. There were no grants on UAB reported before 2007. CURE-UAB, Congress of Urologic Research and Education on Aging UnderActive Bladder. 


\section{Impact on Science}

To determine the impact on science, PubMed was searched for the terms OAB and UAB from 1998 to 2019 (Fig. 1). In the 5 years prior to CURE-UAB (2009-2013), there were 2,391 OAB and $35 \mathrm{UAB}$ references in PubMed. In the 5 years after the first CURE-UAB meeting (2015-2019), there were 2,698 OAB and $204 \mathrm{UAB}$ papers. OAB publications increased by $13 \%$ over the 5 -year period while UAB increased by $483 \%$.

\section{Impact on Research}

Prior to CURE-UAB, there was never a program announcement specific for this disease. After the start of the CURE-UAB series, the NIH issued UAB program announcements in 2015 (PA 15-049, 050, 051) and reissued in 2018 (PA-18-570, 571 and 572: Underactive Bladder and Detrusor Activity in Aging). We then further determined the impact of the CURE-UAB series on research by examining the number of grants related to $\mathrm{UAB}$ or $\mathrm{OAB}$ over time using Grantome.com. Prior to CURE$\mathrm{UAB}$, there were $7 \mathrm{UAB}$ grants ever in total. In the 5 years prior to CURE-UAB (1998-2013), there were 176 OAB and 5 UAB grants, while since the beginning of 2015 , there were $230 \mathrm{OAB}$ and 42 UAB papers (Fig. 1). OAB grants increased by $31 \%$ over the 5 -year period while UAB increased by $740 \%$. Furthermore, the R13 directive leads to the first U.S. Food and Drug Administration (FDA) clinical trial on UAB that is due for completion in 2020 (ClinicalTrials.gov Identifier: NCT02463448).

This display of increase in number of publications and grants on UAB shows the NIH R13 grant mechanism supporting a series of UAB scientific conferences has advanced the awareness of and research on the condition of UAB. A 483\% increase in published research, a $740 \%$ in research grants, and new clinical trials are a direct result from this funding.

\section{PREVALENCE AND NATURAL HISTORY}

The burden of UAB is expected to rapidly rise with the aging population across the world. In the United States alone, individuals over the age of 65 years old will reach 71 million, and will consist of roughly $20 \%$ of the population, by the year 2030 [6]. A recent study highlighted the levels of prevalence and lack of awareness of UAB in the general population - $23 \%$ of people reported having a problem emptying the bladder completely, yet only $11 \%$ had knowledge of UAB [7]. UAB may be due to myogenic causes with impaired contractility due to structural changes of the detrusor muscle and/or neurogenic changes with axonal degeneration demonstrated in unobstructed, aging human bladders $[3,5]$.

The long-term effects of UAB may lead to significant complications. Urine left behind in the bladder can lead to urinary tract infections (UTIs) which, if become chronic, can lead to kidney damage and blood infections. In severe cases, urine left behind in the bladder can build up to a level that causes reflux up the ureters and may also cause kidney damage. Additionally, sediments can accumulate in the bladder, forming bladder stones.

$\mathrm{UAB}$ patients using catheters to empty their bladders also can face long-term medical difficulties, including inflammation and discomfort, the potential for injury, and an increased risk of bacterial infection. For many individuals, the use of catheters can also be a cause for embarrassment and can negatively impact their work and home life. For older patients, it can have a major impact on QoL. The loss of bladder control is the second most common reason for nursing home placement of the elderly [3]. Finding a way to address and treat these bladder issues would allow millions more older adults to remain independent and experience life without the worry and embarrassment associated with bladder control dysfunction.

\section{INNOVATION}

Chronic urinary retention is defined as an elevated postvoid residual (PVR) greater than $300 \mathrm{~mL}$ that persists for at least 6 months and is documented on 2 or more separate occasions. Chronic urinary retention may be caused by either UAB, bladder outlet obstruction (BOO), or both. The AUA Quality Improvement \& Patient Safety Committee proposes that chronic urinary retention be categorized by identifiable high-risk factors and by degree of symptoms. Appropriate follow-up and treatment are based on these stratifications. Four outcome measurements are recommended to be incorporated into future chronic urinary retention treatment studies: assessment of symptoms, reduction of risk, ability to void without catheterization, and stability or progression of symptoms/risk over time [8].

\section{UNDERACTIVE BLADDER APPROVED PRODUCTS}

\section{Bethanechol Chloride}

Bethanechol chloride (common brand names include Urecholine, Duvoid, Myotonachol, and Urocarb) is a synthetic parasympathomimetic that has been widely available since the 
1970s to treat urinary retention. The initial study to describe bethanechol chloride to treat urinary retention was a singlecenter, double-blind study of 20 adults with "decompensated bladder" [9]. The primary outcome reported was "detrusor reaction" in patients with urinary retention, as measured by modified cystometry on the detrusor stretch.

Bethanechol chloride dosage must be individualized, varying with the type and severity of the condition to be treated. The maximal effects of the oral dose of Bethanechol chloride appear within 60 to 90 minutes. Bethanechol chloride can also be administered via subcutaneous injection at lower doses for a quicker response, but then possible adverse reactions must be monitored in the patient. Atropine can abolish the effects of Bethanechol chloride. Bethanechol chloride should not be used when the strength or integrity of the gastrointestinal or bladder wall is in question or when obstructed. At CURE-UAB, a panel of experts reviewing the current available data concluded that there is little beneficial effect of other parasympathomimetic agents in treating or preventing $\mathrm{UAB}[10]$.

\section{Sacral Neuromodulation}

Interstim (Medtronic, Minneapolis, MN, USA) is a sacral neuromodulation device approved by the FDA in 1999 (Premarket Approval P97004/S4) for the indication of refractory, nonobstructive urinary retention. The primary efficacy variable in the retention study population was the output volume per catheterization. Secondary outcomes included analysis of a voiding diary, urodynamics, and QoL assessment by the SF-36 Health Survey and Beck Depression Inventory [11].

The prospective, multicenter trial included randomization to either a treatment group (immediate implant) or control group (delay to implant) [12]. Inclusion criteria required patients to demonstrate $\geq 50 \%$ reduction in catheter volume during percutaneous test stimulation compared to baseline (no stimulation). Sixty-eight patients met criterion for randomization. Results at 6-month follow-up revealed a significant reduction in catheterization volume $(\mathrm{P}<0.001)$ in implant compared to control patients. Control patients $(n=22)$ had an average catheterization volume of $350 \pm 152 \mathrm{~mL}$ at baseline and in 6 months an average catheterization volume of $319 \pm 195 \mathrm{~mL}$. Patients in the treatment arm (implant group) had an average catheterization volume at baseline of $339 \pm 176 \mathrm{~mL}$ and 6 months posttreatment had an average catheterization volume of $49 \pm 106 \mathrm{~mL}$. Additionally, $69 \%$ of implants (20 of 29) stopped catheterization while only $9 \%$ of controls ( 2 of 22 ) stopped catheterization.

\section{InFlow Intraurethral Valve-Pump Medical Device}

The most recent FDA approved product is the InFlow Intraurethral Valve-Pump device (Vesiflo, Redmond, WA, USA) for use in women with incomplete bladder emptying due to neurogenically impaired detrusor contractility. Approved in 2015, the InFlow is a non-surgical urinary prosthesis intended to provide bladder drainage for women with $\mathrm{UAB}$ [13] and is replaced monthly (every 29 days or less).

The initial clinical trial enrolled 273 adult women with impaired detrusor contractility that were using CIC and were capable of operating the device. In 98\% of subjects (113 of 115) with PVR data available for both baseline and treatment, the median treatment PVR was no greater than the median baseline PVR. A key secondary endpoint was QoL measurement. Among those subjects with both baseline and treatment QoL data, patients using InFlow increased their QoL scores by an average of 25 points on a 100 points scale $(\mathrm{P}<0.0001)$. The median percent improvement was $54 \%$. The results were both statistically and clinically significant. However, this device may not be ideal for all patients as during the trial, more than half of the women stopped using inFlow due to discomfort and/or leakage of urine.

\section{UNDERACTIVE BLADDER INVESTIGATIONAL PRODUCTS}

\section{Autologous Muscle-Derived Cells for UAB}

Given that degeneration or loss of detrusor smooth muscle is regarded as one of the contributors to UAB associated with aging, regenerative medicine is now in clinical trial for UAB. The first step is collecting and harvesting Autologous muscle-derived cells (AMDCs) by muscle biopsy. AMDCs are purified and expanded by an external specialized lab (Cook MyoSite, Inc., Pittsburgh, PA, USA). Lastly, they are shipped back to the clinic where they are then injected into the detrusor of enrolled patients. Skeletal muscle-derived cells injected into the bladder wall have shown evidence of having a smooth muscle phenotype, inducing innervation, and having enhanced detrusor contraction as measured by urodynamics [14]. An initial compassionate-use case demonstrated feasibility and safety at Beaumont Health System in Royal Oak, Michigan. This led to an ongoing clinical trial. This is a 1-year prospective open-label study assessing the safety and efficacy of intradetrusor injected AMDC treatment for UAB [15] (ClinicalTrial.gov NCT02463448). Diokno et al. [16], at a late-breaking plenary session of the 2019 
AUA meeting (Chicago, IL, USA) presented promising interim data on this first regulatory approved clinical trial.

As of November 2019, twenty nonneurogenic UAB patients were treated and 16 had a 12-month follow-up visit. Baseline evaluation included history, physical, voiding diary, multichannel urodynamic testing, and endoscopy to confirm no obstruction. Key inclusions included difficult bladder emptying symptoms, Underactive Bladder Questionnaire Score of 3+/or be on total CIC, unresponsive to therapy, PVR of $150 \mathrm{~mL}$ or more, and no evidence of obstruction. Key exclusions included neurological impairment, pelvic organ cancers, pelvic radiation therapy, and currently on medications affecting bladder.

Approximately $150 \mathrm{mg}$ of quadriceps femoris muscle was collected using a spirotome 8-G needle, sent to Cook MyoSite, processed and upon reaching a final concentration of 62.5 million $\mathrm{AMDC} / \mathrm{mL}$, the patient received 30 intradetrusor injections stem cells delivered to the bladder detrusor. This procedure is performed utilizing a flexible cystoscope under direct vision using topical local anesthesia. Follow-up assessments included adverse events (AEs) and efficacy at 1-, 3-, 6-, 12-month postinjection. An optional second injection was offered at the end of 6-month visit.

The study enrolled 20 subjects, including 15 men and 5 women. Ages range from 41-82, with a median age of 65 years old. The etiology included 7 men with benign prostatic hyperplasia after transurethral resection of the prostate, and 13 women and men with idiopathic UAB. AMDC was injected once in 20 subjects and injected twice in 17 subjects. Bladder function at baseline included CIC-only in 10 subjects, voiding only in 1 subject, and mixed CIC/voiding in 9 subjects.

Preliminary data results on 15 subjects showed a gradual increase in the number of subjects reporting improvement in UAB symptoms over time. This was based on participants' reports by a Global Response Assessment (GRA) and collaborated by 3-day voiding diaries. At 1-, 3-, 6-, and 12-month followup visits, $27 \%$ ( 4 of 15), 31\% (4 of 13), 33\% (4 of 12), and 70\% (7 of 10) reported an overall improvement by GRA respectively. Improved patients reported decreased hesitancy and straining, with improved emptying sensation. No patients reported worse symptoms by GRA during this entire period. No serious procedure or treatment-related AEs occurred. This interim analysis suggests intradetrusor $A M D C$ for $\mathrm{UAB}$ is safe and for some patients will improve bladder function. Cellular therapy may be a promising novel treatment for $\mathrm{UAB}$ and a multicenter-controlled trial is needed.

\section{Neurokinin2 receptor agonists}

A Neurokinin-2 receptor (NK2R) agonists are being developed by Dignified as an "on-demand, rapid-onset, short duration, drug-induced, voiding therapy" for patients that require catheterization and do not have BOO [17]. The lead candidate is DTI-100, a short amino acid peptide analog of neurokinin A. DTI-100 produces highly efficient voiding in under 5 minutes of intravenous or subcutaneous administration in $>85 \%$ of awake preclinical models including rats, dogs, minipigs, and monkeys. In anesthetized animals, DTI-100 administration produces rapid ( $<2$ minutes), short duration (10 minutes), powerful contractions of both the bladder $(>40 \mathrm{~mm} \mathrm{Hg}$ ) as well as the rectum ( $>30 \mathrm{~mm} \mathrm{Hg}$ ). All effects on the bladder and rectum can be blocked by NK2R antagonist GR159897. Vomiting, gastrointestinal discomfort, and hypotension were observed at higher doses and are expected to be the dose-limiting side effect in clinical trials. Although preclinical studies have not yet detected clinically significant increases in bladder pressure during voiding cystometry or under bladder isovolumetric cystometry conditions in acute injury spinal rats, voiding pressures were briefly elevated (up to $60 \mathrm{~mm} \mathrm{Hg}$ ) in anesthetized rats without spinal cord injury [17].

Peptide NK2R agonists may provide an on-demand voiding therapy as it acts almost instantly yet lasts only for a short duration, thereby allowing an individual to control when micturition occurs and reduces or eliminates the need for catheterization. Potential unwanted side effects, the effects on the bowel, the presence of $\mathrm{BOO}$, and spinal cord injury may need to be considered in its clinical utilization.

\section{ASP8302}

A muscarinic M3 receptor modulator, ASP8302, by Astellas Pharma is a under phase 2 development for UAB [18] (ClinicalTrial.gov NCT03702777). The M3 cholinergic receptor is responsible for parasympathetic detrusor contraction and is found mainly in the smooth muscles and glands of the bladder. The objectives of this study are to evaluate the efficacy, pharmacokinetics, safety and tolerability of ASP 8302 capsules orally compared with placebo in participants with UAB. The multicenter study will have estimated enrollment of 130 adult men and women in Europe and Japan, comprised of a screening visit followed by a treatment period and a 2-week follow-up period. The primary outcome measures the change in PVR from baseline to treatment week 6 after standardized bladder filling measured by catheterization (PVRc2). Secondary outcomes mea- 
sure the voided volume after standardized bladder filling. Bladder voiding efficiency is then calculated using PVRc2 and standardized bladder filling.

Inclusion criteria include male and female over 18 years old with a UAB diagnosis, defined as a bothersome chronic incomplete bladder emptying for $\geq 6$ months before screening with a PVR $\geq 75 \mathrm{~mL}$ (measured by ultrasound after uroflowmetry). Exclusion criteria are extensive for other urologic conditions, other medications, and other concomitant conditions [18]. Subjects on CIC should be on CIC for at least 1 month and should still be able to void spontaneously. The estimated primary completion date is June 2020 . It should be noted that due to the extensive distribution of muscarinic receptors in the body, there may be a broad spectrum of adverse effects from anticholinergic medications which may include salivation/drooling, loss of accommodation, dizziness, headache, and ileus.

\section{TAC-302}

Taiho Pharmaceutical Co's TAC-302 is a cyclohexenoic fatty alcohol derivative that can promote neurite outgrowth in isolated peripheral neurons and prevents bladder denervation related bladder dysfunctions following BOO in rats $[19,20]$. In preclinical studies, TAC-302 improved storage and voiding dysfunctions by improving bladder denervation and detrusor underactivity (DU) even when the treatment was started after storage and voiding dysfunctions had already occurred [21].

The current phase 2 study (ClinicalTrials.gov NCT03175029) enrolled 75 male and female adult subjects to TAC-302 or placebo [19]. The main purpose of this study is to assess the efficacy of TAC-302 for 12 weeks in DU patients with OAB by measuring the following parameters of pressure-flow study: the bladder contractility index (BCI) in males and the projected isovolumetric pressure (PIP) in females. Pressure-flow study parameters of the detrusor pressure at maximum flow rate and the maximum flow will be combined to report BCI or PIP.

Inclusion criteria include lower urinary tract symptoms for at least 12 weeks prior to study entry, at least 1 urinary urgency episodes per day, and diurnal urinary frequency of 8 or more per day, and meet the DU criteria by urodynamic study. Exclusion criteria for this study are patients with neurogenic bladder by the central nervous system diseases, stage III or more cystocele of pelvic organ prolapse quantification system (women), prostate volume $\geq 30 \mathrm{~mL}$ (men), and any symptoms of UTI.

\section{Bladder Scanner for the Patient and Caregiver}

Measurement of PVR is largely accepted as a primary measurement for the diagnosis and follow-up of UAB. There is an increased risk of UTI in UAB patients with large PVR, therefore CIC should be avoided when possible. As such, the use of ultrasound should be the standard-of-care. Recent technology progress is being made towards making ultrasound convenient, inexpensive, and that can be done at the point-of-care. In 2015, a bladder residual volume scanner received regulatory approval in Japan.

The Lilium a-200 (Lilium Otsuka Co., Ltd., Sagamihara, USA) is a continuous, noninvasive bladder urine volume sensor. It is a hand-held urine volume sensor which measures the residual bladder volume. The small size and simplicity of the scanner may allow for better urination or CIC schedules, and may monitor catheterization volume in patients with UAB or those with diminished sensation of bladder filling. The device is planned to seek regulatory approval and introduction in the United States in the near future.

\section{CASE STUDY OF ONE PRESENTATION AND PUBLICATION FROM CURE-UAB: OAB TO OAB HYPOTHESIS}

One presentation and paper from the first CURE-UAB meeting that has often been referenced is the "OAB to UAB Chancellor Hypothesis." It states that "Patients with chronic untreated or

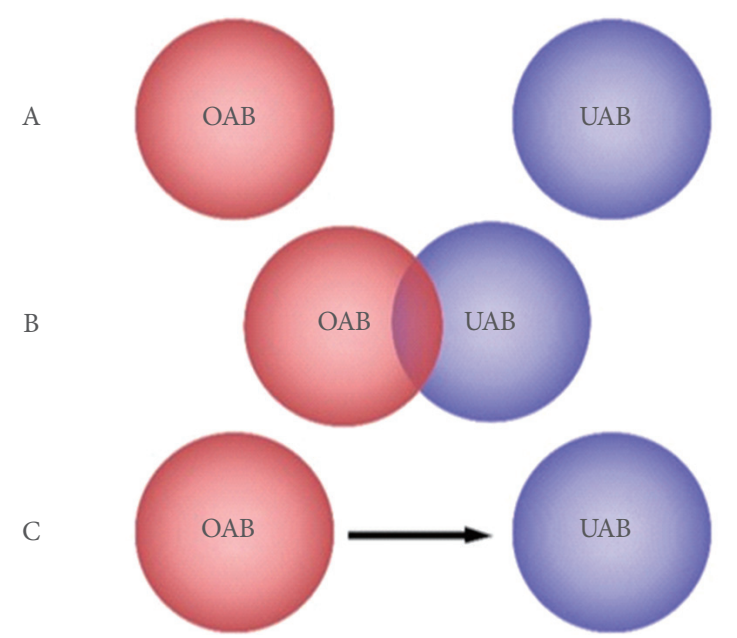

Fig. 2. Several ways to consider the relationship between overactive bladder $(\mathrm{OAB})$ and underactive bladder (UAB). (A) OAB and $\mathrm{UAB}$ as separate conditions. (B) OAB and $\mathrm{UAB}$ as overlapping syndromes. (C) $\mathrm{OAB}$ may progress to $\mathrm{UAB}$. 
treatment refractory $\mathrm{OAB}$ have a significant risk of progression to UAB" [22]. The purpose of this hypothesis is to stimulate basic and clinical research on UAB to provide an effective treatment for patients suffering with $U A B$ and reduce the risk of the development of $\mathrm{UAB}$ in $\mathrm{OAB}$ patients. A brief summary of the presentation and hypothesis is below.

Both $\mathrm{OAB}$ and $\mathrm{UAB}$ are common in older patients, and both syndromes share common symptoms including frequency and nocturia. Both can present together in the same patient, referred to as detrusor hyperreflexia impaired contractility (DHIC), and both are associated with common underlying etiologies such as BOO and neurological diseases (Fig. 2) [22].

Similar to how impaired bladder emptying is associated with older men and women, sarcopenia also increases with aging [23]. Impaired bladder emptying is often associated with detrusor overactivity with or without BOO [24]. DHIC is a common and important finding based on urodynamic studies in both healthy and symptomatic elderly. Griffiths et al. noted the coincidental occurrence and overlap of $\mathrm{OAB}$ and $\mathrm{UAB}$ with different etiological factors in patient populations while histological studies detected age-related change in the bladder associated with both detrusor overactivity and underactivity in DHIC patients [25].

Chronic muscular overactivity in $\mathrm{OAB}$ may lead to muscle fatigue or progressive ischemia, inflammation, and oxidative damage, which can lead to hypocontractility. Research has demonstrated that bladder wall thickens and increases in mass in patients with $\mathrm{OAB}[26,27]$ and that urine nerve growth factor levels escalate [28]. OAB might involve changes that could eventually lead to alterations in muscle, connective tissue structure, and ultimately bladder function, resulting in impaired contractility. In many patients, $\mathrm{OAB}$ symptoms improve after BOO treatment [1]. However, if BOO is not treated UAB may develop as a late consequence.

Ischemia may be an important etiology of UAB. High pressure is generated by the overactive detrusor, resulting in urine release. Following urine release, even a small amount, triggers the subsequent relaxation of the detrusor and production of free radicals within the bladder. These free radicals insult the detrusor, which over time can cause permanent damage to the detrusor resulting in deterioration of normal bladder function [29]. With this mechanism in mind, for the mechanism of chronic obstruction could result in a similar etiology. As with reperfusion injury, every time the patient voids and generates high intravesical pressure, transient ischemia is caused and with

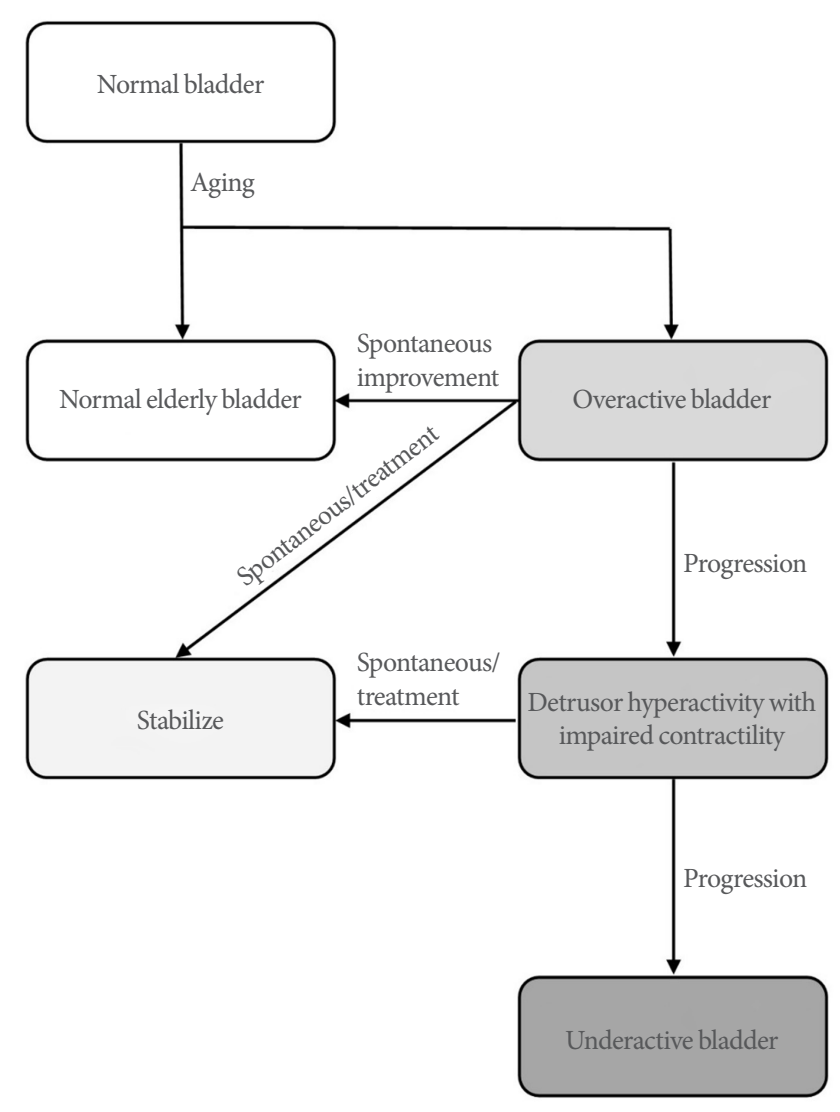

Fig. 3. The overactive bladder $(\mathrm{OAB})$ to underactive bladder (UAB) hypothesis: patients with chronic untreated or treatment refractory $\mathrm{OAB}$ have a significant risk of progression to $\mathrm{UAB}$.

pressure release during voiding free radicals or injurious substances may be released, causing cumulative injury to the detrusor. Perhaps chronic pressure and hypoxia-induced pressure can be initially compensated with $\mathrm{OAB}$, but decompensation in those that manifest as DHIC, ultimately leading to UAB (Fig. 3).

\section{CONCLUSIONS}

$\mathrm{UAB}$ is one of the greatest areas of unmet need and yet has the greatest opportunity for advancement in functional urology. The disease is common, severe, and lacks effective treatment. There is great international interest in the research of $\mathrm{UAB}$ and we believe the path forward should include engaging regulatory agencies around the world that can harmonize and formalize guidance for regulatory trial designs for therapeutics for UAB. The CURE-UAB conference series as sponsored by the United States National Institute of Health has helped raise awareness and our knowledge of $\mathrm{UAB}$, as well as increasing $\mathrm{UAB}$ research. 
Understanding the natural history of the disease, identifying individuals at risk for further deterioration, and exploring new therapies are top research priorities. All stakeholders, including physicians, nurses, basic science researchers, patient advocates, and government policy makers, are needed to improve patient outcomes as well as public awareness on UAB.

\section{ACKNOWLEDGEMENTS}

The authors would like to acknowledge the NIA, NIDDK, the Underactive Bladder Foundation (www.underactivebladder. org), and the Aikens Center for Neurourology Research at Beaumont Health System (Royal Oak, MI). We would also like to thank all the participants of CURE-UAB meetings for their insights, questions, and sharing their research.

The views expressed in written conference materials or publications and by speakers and moderators do not necessarily reflect the official policies of the NIH; nor does mention by trade names, commercial practices, or organizations imply endorsement by the U.S. Government.

\section{AUTHOR CONTRIBUTION}

- Conceptualization: $M B C, A D$

- Formal Analysis: SNB, LEL, EW, BZ

- Investigation: $A D, M B C, E W$

- Methodology: $A D$

- Writing - Original Draft: $M B C, S N B, L E L, E W$

-Writing - Review \& Editing: $M B C, A D, S N B, L E L, E W, B Z$

\section{REFERENCES}

1. van Koeveringe GA, Vahabi B, Andersson KE, Kirschner-Herrmans R, Oelke M. Detrusor underactivity: a plea for new approaches to a common bladder dysfunction. Neurourol Urodyn 2011;30:723-8.

2. Osman NI, Chapple CR, Abrams P, Dmochowski R, Haab F, Nitti $\mathrm{V}$, et al. Detrusor underactivity and the underactive bladder: a new clinical entity? A review of current terminology, definitions, epidemiology, aetiology, and diagnosis. Eur Urol 2014;65:389-98.

3. Chancellor MB, Diokno AC. The underactive bladder. Cham (Switzerland): Springer International Publishing; 2016.

4. Chancellor MB, Bartolone SN, DeVries EM, Diokno AC, Gibbons $\mathrm{M}$, Jankowski R, et al. New technology assessment and current and upcoming therapies for underactive bladder. Neurourol Urodyn
2018;37:2932-7.

5. Dewulf K, Abraham N, Lamb LE, Griebling TL, Yoshimura N, Tyagi $\mathrm{P}$, et al. Addressing challenges in underactive bladder: recommendations and insights from the Congress on Underactive Bladder (CURE-UAB). Int Urol Nephrol 2017;49:777-85.

6. Chuang YC, Plata M, Lamb LE, Chancellor MB. Underactive bladder in older adults. Clin Geriatr Med 2015;31:523-33.

7. Valente S, DuBeau C, Chancellor D, Okonski J, Vereecke A, Doo F, et al. Epidemiology and demographics of the underactive bladder: a cross-sectional survey. Int Urol Nephrol 2014;46 Suppl 1:S7-10.

8. Stoffel JT, Peterson AC, Sandhu JS, Suskind AM, Wei JT, Lightner DJ. AUA White Paper on nonneurogenic chronic urinary retention: consensus definition, treatment algorithm, and outcome end points. J Urol 2017;198:153-60.

9. Diokno AC, Lapides J. Action of oral and parenteral bethanechol on decompensated bladder. Urology 1977;10:23-4.

10. Krishnamoorthy S, Kekre NS. Detrusor underactivity: to tone or not to tone the bladder? Indian J Urol 2009;25:407-8.

11. Jonas U, Fowler CJ, Chancellor MB, Elhilali MM, Fall M, Gajewski JB, et al. Efficacy of sacral nerve stimulation for urinary retention: results 18 months after implantation. J Urol 2001;165:15-9.

12. U.S. Food and Drug Administration. Premarket approval supplement number P970004/S4. Implantable Electrical Stimulator for Incontinence [Internet]. Silver Spring (MD): U.S. Food and Drug Administration; 2019 [cited 2019 Dec 7]. Available from: https://www.accessdata.fda.gov/scripts/cdrh/cfdocs/cfpma/pma.cfm?ID=P970004.

13. U.S. Food and Drug Administration. De novo classification request for inflow intraurethral valve-pump and activator [Internet]. Silver Spring (MD): U.S. Food and Drug Administration; 2013 Oct 25 [cited 2019 Dec 7]. Available from: https://www.accessdata.fda.gov/ cdrh_docs/reviews/DEN130044.pdf.

14. Huard J, Yokoyama T, Pruchnic R, Qu Z, Li Y, Lee JY, et al. Musclederived cell-mediated ex vivo gene therapy for urological dysfunction. Gene Ther 2002;9:1617-26.

15. ClinicalTrials.gov. Autologous muscle derived cells for underactive bladder [Internet]. Bethesda (MD): ClinicalTrials.gov; 2015 [cited 2019 Dec 7]. Available from: https://clinicaltrials.gov/ct2/show/ NCT02463448.

16. Diokno AC, Gilleran J, Sirls L, Hasenau D, Bowlus J, Shea E, et al. LBA-04; intradetrusor injection of adult muscle-derived cells for the treatment of underactive bladder: interim report on safety and efficacy (NCT02463448). J Urol 2019;201(Suppl 4):e993.

17. Kullmann FA, Katofiasc M, Thor KB, Marson L. Pharmacodynamic evaluation of Lys5, MeLeu9, Nle10-NKA(4-10) prokinetic effects on bladder and colon activity in acute spinal cord transected and 
spinally intact rats. Naunyn Schmiedebergs Arch Pharmacol 2017; 390:163-73.

18. ClinicalTrials.gov. A study of ASP8302 in participants with underactive bladder [Internet]. Bethesda (MD): ClinicalTrials.gov; 2018 [cited 2019 Dec 7]. Available from: https://clinicaltrials.gov/ct2/ show/NCT03702777.

19. ClinicalTrials.gov. Exploratory study of TAC-302 in detrusor underactivity patients with overactive bladder [Internet]. Bethesda (MD): ClinicalTrials.gov; 2017 [cited 2019 Dec 7]. Available from: https://clinicaltrials.gov/ct2/show/NCT03175029.

20. Yoshida S, Orimoto N, Tsukihara H, Noma T, Hakozaki A, Sasaki E. TAC-302 promotes neurite outgrowth of isolated peripheral neurons and prevents bladder denervation related bladder dysfunctions following bladder outlet obstruction in rats. Neurourol Urodyn 2018;37:681-9.

21. Yoshida S, Noma T, Miyoshi K, Tsukihara H, Orimoto N, Hakozaki A, et al. Therapeutic effect of TAC-302, a cyclohexenoic fatty alcohol derivative, on bladder denervation-related storage and voiding dysfunctions in rats. Neurourol Urodyn 2018;37:2106-13.

22. Chancellor MB. The overactive bladder progression to underactive bladder hypothesis. Int Urol Nephrol 2014;46 Suppl 1:S23-7.

23. Cucchi A, Quaglini S, Rovereto B. Development of idiopathic de- trusor underactivity in women: from isolated decrease in contraction velocity to obvious impairment of voiding function. Urology 2008; 71:844-8.

24. Resnick NM, Yalla SV. Detrusor hyperactivity with impaired contractile function. An unrecognized but common cause of incontinence in elderly patients. JAMA 1987;257:3076-81.

25. Griffiths D, Tadic SD, Schaefer W, Resnick NM. Cerebral control of the bladder in normal and urge-incontinent women. Neuroimage 2007;37:1-7.

26. Khullar V, Salvatore S, Cardozo L, Bourne TH, Abbott D, Kelleher C. A novel technique for measuring bladder wall thickness in women using transvaginal ultrasound. Ultrasound Obstet Gynecol 1994;4:220-3.

27. Ukimura O, Kojima M, Iwata T, Inaba M, Miki T. Ultrasonic measurement of bladder weight as a novel urodynamic modality. Adv Exp Med Biol 2003;539(Pt A):311-5.

28. Kim JC, Park EY, Seo SI, Park YH, Hwang TK. Nerve growth factor and prostaglandins in the urine of female patients with overactive bladder. J Urol 2006;175:1773-6; discussion 1776.

29. Finkbeiner A, Lapides J. Effect of distension on blood flow in dog's urinary bladder. Invest Urol 1974;12:210-2. 